\title{
Strain, alloy composition, and lattice relaxation measured by optical-absorption spectroscopy
}

\author{
S. A. Clark, ${ }^{a)}$ P. Roura, J. Bosch, A. Pérez-Rodríguez, and J. R. Morante \\ LCMM, Departament de Física Aplicada i Electrónica, Facultat de Física, Universitat de Barcelona, \\ Diagonal 645, E-08028 Barcelona, Catalonia, Spain \\ D. I. Westwood and R. H. Williams \\ Department of Physics and Astronomy, University of Wales, College of Cardiff, P.O. Box 913 , \\ Cardiff, CF2 3YB. Wales, United Kingdom
}

(Received 22 June 1994; accepted for publication 7 December 1994)

\begin{abstract}
The validity of optical absorption (OA) as a technique for the measurement of strain $e_{11}$, alloy composition $x$, and relaxation in $\mathrm{In}_{x} \mathrm{Ga}_{1-x}$ As epilayers on $\mathrm{InP}$ has been examined by comparison with similar measurements by double-crystal x-ray diffraction (DCXD). Provided that the strain arising from differences in the thermal contraction of the substrate and epilayer are taken into account, measurements of strain by OA show good agreement with DCXD results, with a dispersion of $\Delta e_{11}= \pm 0.27 \times 10^{-3}$. Comparison of alloy compositions given by the two techniques shows similarly good agreement, with a dispersion in the values of $x$ of less than $\Delta x= \pm 0.7 \%$. OA may also be used to determine lattice relaxation. The degree of uncertainty in the measurement of this parameter increases as lattice match is approached and decreases as the lattice relaxes. Our studies indicate that $\mathrm{OA}$ may be used as an independent technique to evaluate strain, alloy composition, and the degree of lattice relaxation in $\mathrm{In}_{x} \mathrm{Ga}_{1-x}$ As epilayers. (1) 1995 American Institute of Physics.
\end{abstract}

\section{INTRODUCTION}

Epitaxial structures of low dimensions are of high technological interest for a wide variety of applications including optical telecommunications, optical computing, and quantum electronics. A new degree of freedom in the tailoring of the valence-band structure has recently been achieved by the pseudomorphic growth of mismatched materials. Biaxial strain splits the degeneracy of the light-hole (LH) and heavyhole $(\mathrm{HH})$ center states, leading to the possibility of the highest band being either $\mathrm{LH}$ - or HH-like.

The ternary material $\operatorname{In}_{x} \mathrm{Ga}_{1-x}$ As, when grown on InP(100) substrates, experiences a strain whose value depends on the In content $x$. At room temperature $\operatorname{In}_{x} \mathrm{Ga}_{3-x}$ As is lattice matched to InP at $x=53.2 \%$. At other values of $x$, assuming that the lattice is deformed from cubic to tetragonal, with lattice parameters $a_{\|}$and $a_{\perp}$, parallel and perpendicular to the interface respectively, the strain $e_{11}$ induced in the plane of the interface is given by

$$
e_{11}=\frac{a_{\|}-a_{0}}{a_{0}}
$$

where $a_{0}$ is the lattice parameter of the completely relaxed epilayer. Measurements of $e_{11}, x$, and lattice relaxation are commonly made using structural techniques such as doublecrystal $\mathrm{x}$-ray diffraction (DCXD). ${ }^{1}$ However, as band engineering is the prime interest in these materials, optical techniques can offer the advantage of the direct measurement of the band gap and valence-band splitting in relation to these parameters. In this study, we describe a method for using optical absorption (OA) as an alternative and independent technique for the measurement of strain, alloy composition,

"Current address: Division of Applied Physics, Sheffield Hallam University, Pond St., Sheffield S1 IWB, United Kingdom. and relaxation in $\mathrm{In}_{x} \mathrm{Ga}_{1}-x$ As epilayers on InP. The validity of the technique has been assessed by a systematic comparison of OA and DCXD measurements of these parameters, obtained from a series of $\mathrm{In}_{x} \mathrm{Ga}_{1-x}$ As epilayers, grown on InP(100) by molecular-beam epitaxy (MBE).

\section{EXPERIMENTAL DETAILS}

All the $\operatorname{In}_{x} \mathrm{Ga}_{1-x}$ As layers were grown in a VG Semicon V80H MBE system. Growth was carried out at $515^{\circ} \mathrm{C}$ on InP $(001) \pm 0.50^{\circ}$, semi-insulating Fe-doped substrates. The thicknesses of the layers ranged from 0.4 to $2.0 \mu \mathrm{m}$, grown at a rate of $1 \mu \mathrm{m} \mathrm{h}^{-1}$. In/Ga fluxes were adjusted to grow epilayers with compressive $(x>53.2 \%)$ and also tensile strains $(x<53.2 \%)$, in the range $47 \%<x<57 \%$. The layers were not intentionally doped, although Hall-effect measurements showed all the layers to be $n$ type, with residual carrier concentrations in the range $n=(1-3) \times 10^{16} \mathrm{~cm}^{-3}$.

$\mathrm{X}$-ray-diffraction measurements were performed using a Bede 150 double-crystal diffractometer. Rocking curves were taken for the symmetric (004) and asymmetric (115) glancing incidence and glancing exit reflections. The inplane strain, alloy composition, and relaxation of the epilayers were calculated following a method that has been described previously. ${ }^{1}$

The OA spectra were obtained using a Fourier transform interferometer; the spectral resolution was $0.1 \mathrm{meV}$. The samples were illuminated by a tungsten lamp with a $\mathrm{CaF}_{2}$ beam splitter and the transmitted light was detected using a cooled InSb detector. The use of a continuous-flow He cryostat facilitated the measurement of $\mathrm{OA}$ spectra as a function of sample temperature in the range from 4 to $200 \mathrm{~K}$. 


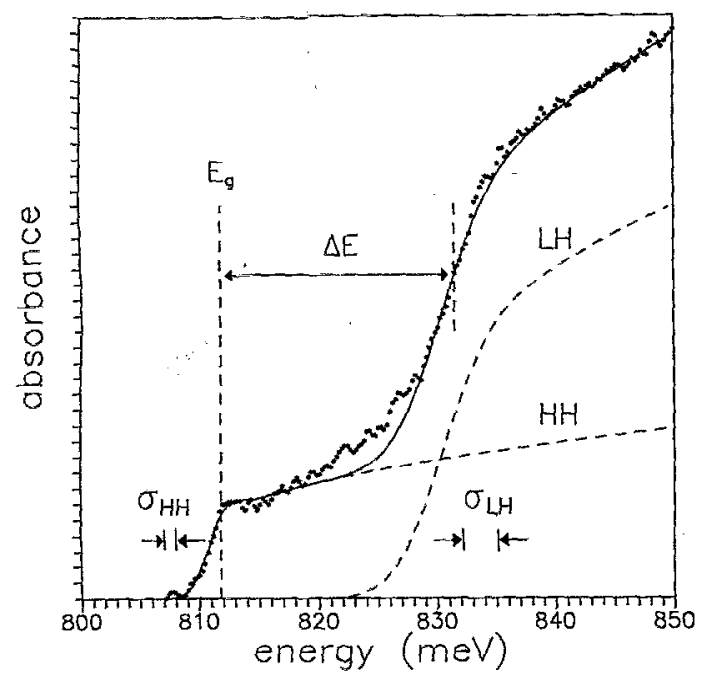

FIG. 1. Oplical-absurptiun spectra obtained at $4 \mathrm{~K}$ from sample $\mathrm{B}$ (tensile). The calculated spectrum is shown as a continuous line and the dashed lines denote the contributions from the LH and HH. The calculated band gap $E_{g}$ and valence-band splitting are also shown.

\section{SPECTRAL FITTING}

In Figs. 1 and 2, typical OA spectra obtained from $\mathrm{In}_{x} \mathrm{Ga}_{1-x}$ As epilayers are shown; both spectra exhibit a splitting in the valence-band edge arising from an internal strain in the epilayer. Band-structure calculations give an optical matrix element between conduction and valence bands that is more intense in the case of LH than HH subbands. ${ }^{2}$ For tensile layers the $\mathrm{HH}$ band determines the band gap; we can thus infer that the epilayer in Fig. 1 is subjected to tensile strain. Conversely, in Fig. 2, the band gap is determined by the more intense LH band, indicating that the strain is compressive.

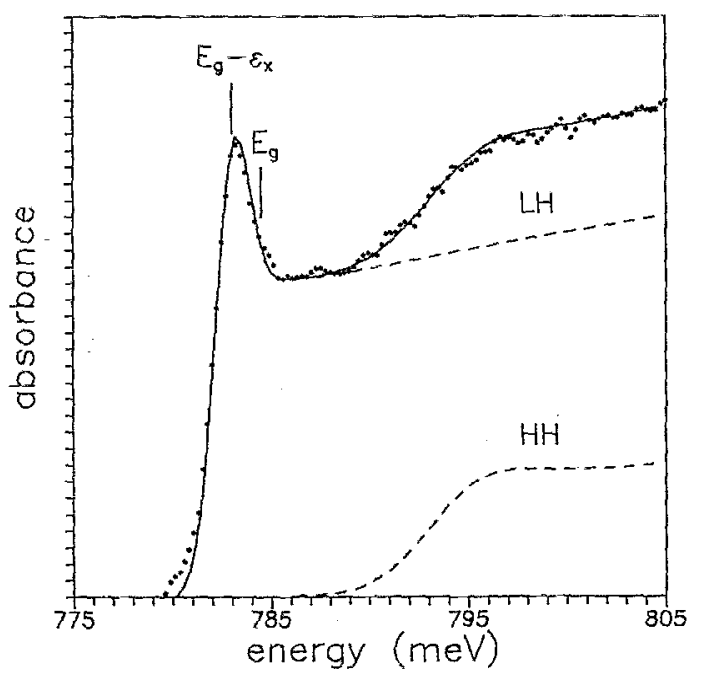

FIG. 2. Optical-absorption spectra obtained at $4 \mathrm{~K}$ from sample $\mathrm{G}$ (compressive). The calculated spectrum is shown as a continuous line and the dashed lines denote the contributions from the $\mathrm{LH}$ and $\mathrm{HH}$. The calculated band gap and the exciton energy $\epsilon_{x}$ are also shown.
To obtain accurate values of band parameters, all the OA spectra were analyzed using a curve-fitting routine based on Elliot's formula. ${ }^{3}$ For nondegenerate parabolic bands, the absorption coefficient $\alpha$ is described by

$$
\begin{gathered}
\alpha(h \nu)=\frac{2 \pi \sqrt{\epsilon_{x}}}{h \nu}\left[\sum_{n=1}^{\infty} \frac{2 \epsilon_{x}}{n^{3}} \delta\left(h \nu-E_{g}+\frac{\epsilon_{x}}{n^{2}}\right)\right. \\
\left.+\frac{H\left(h \nu-E_{g}\right)}{1-\exp \left(-2 \pi \sqrt{\left.\epsilon_{x} / h \nu-E_{g}\right)}\right.}\right],
\end{gathered}
$$

where $E_{g}$ is the band-gap energy, $\epsilon_{x}$ is the exciton binding energy, $H$ is the Heavyside function, and $A$ is a constant that contains the optical matrix element between the atomic wave functions. The first term is the absorption due to the discrete bound states of the exciton and the second is due to the continuum. The valence-band splitting $\Delta E$ is included by adding the contributions of the $\mathrm{HH}$ and $\mathrm{LH}$ subbands, each one of which has the spectral shape of Eq. (2). To account for microscopic inhomogeneities, the contributions to the spectrum from the two bands were convoluted with Gaussian distributions, characterized by standard deviations $\sigma_{\mathrm{HH}}$ and $\sigma_{\mathrm{LH}}$. The calculated spectra are also shown in Figs. 1 and 2; the L.H and HH contributions are shown to illustrate the internal structure of the spectra. The parameters adjusted to fit the experimental spectra are the following.

(i) The amplitudes of the $\mathrm{HH}$ and $\mathrm{LH}$ subbands, $A_{\mathrm{HH}}$ and $A_{\mathrm{LH}}$, from which, with calibration, a measure of the epilayer thickness may be extracted.

(ii) The spectral dispersion of the LH and HH subbands, $\sigma_{\mathrm{LH}}$ and $\sigma_{\mathrm{HH}}$. Its effect may be visualized in the spectrum as a smoothing of the band edges (see Fig. 1). If the dispersion is small enough, the peak corresponding to the exciton ground state can be resolved (see the LH band in Fig. 2). An indication of the origins of the microscopic inhomogeneities may be deduced from the relative energy dispersion, $\sigma_{\mathrm{HH}} / \sigma_{\mathrm{LH}} \cdot{ }^{4} \mathrm{~A}$ value of $\sigma_{\mathrm{HH}} / \sigma_{\mathrm{LH}}<1$ indicates the preseñce of composition inhomogeneities, whereas $\sigma_{\mathrm{HH}} / \sigma_{\mathrm{LH}} \approx 2.8$ indicates the existence of an inhomogeneous strain field.

(iii) The exciton binding energy $\epsilon_{x}$ (illustrated in Fig. 2). Its effect on the spectral shape is quite complex. If the value of $\epsilon_{x}$ is sufficiently large the exciton peak can be resolved; otherwise, its main effect is to determine the shape of the continuum part of the spectrum.

(iv) The band-gap energy $E_{g}$ and the band-gap splitting energy $\Delta E=E_{g \mathrm{LH}}-E_{g \mathrm{HH}}$. Their values determine the energetic position of the bands (see Fig. 1), but have only a minor influence on the shape of the spectrum. Of prime interest in this study was the extraction of the values of $E_{g}$ and $\Delta E$ for the calculation of $e_{11}, x$, and consequently the relaxation of the epilayer.

From the above description it is clear that each parameter influences the spectral shape quite independently. Thus, although several free parameters are used, we can have great confidence in the fitted values. 


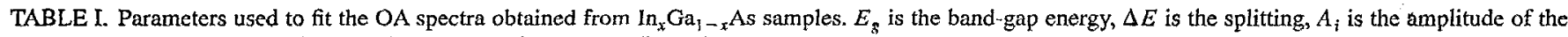
light and heavy-hole subbands, $\sigma_{i}$ their corresponding energy dispersion, and $\epsilon_{x}$ the exciton binding energy.

\begin{tabular}{|c|c|c|c|c|c|c|}
\hline Sample & $E_{g}(\mathrm{meV})$ & $\Delta E(\mathrm{meV})$ & $A_{\mathrm{HH}} / A_{\mathrm{LH}}$ & $\sigma_{\mathrm{L}, \mathrm{H}}(\mathrm{meV})$ & $\sigma_{\mathrm{HH}} / \sigma_{\mathrm{LH}}$ & $\epsilon_{x}(\mathrm{meV})$ \\
\hline A & 824.5 & $\quad 29.5$ & 0.35 & 7.7 & 0.9 & 1.0 \\
\hline $\mathrm{B}$ & 811.8 & 19.7 & 0.35 & 3.2 & 0.3 & 0.6 \\
\hline C & 801.9 & 6.7 & 0.36 & 1.8 & 1.0 & 0.8 \\
\hline D & 804.6 & 5.0 & 0.35 & 1.7 & 0.6 & 0.8 \\
\hline E & 801.0 & -1.1 & 0.35 & 0.8 & 2.0 & 1.2 \\
\hline$F$ & 796.8 & -2.6 & 0.34 & 0.6 & 2.5 & 1.1 \\
\hline$G$ & 784.5 & $=11.4$ & 0.32 & 0.9 & 2.8 & 1.5 \\
\hline
\end{tabular}

\section{RESULTS AND ANALYSIS}

For all the samples examined in this study, the calculated and experimental spectra showed a reasonable fit. The values of the fitting parameters obtained for each sample are summarized in Table I. As in previous studies, the values of $A_{\mathrm{HH}} / A_{\mathrm{LH}}$ obtained lie near $0.35 .^{4}$ From the relative energetic dispersion $\sigma_{\mathrm{HH}} / \sigma_{\mathrm{LH}}$, it is seen that the inhomogeneities of composition are predominant in the tensile samples $\left(\sigma_{\mathrm{HH}} / \sigma_{\mathrm{LH}} \leqslant 1\right)$, whereas an inhomogeneous strain field is present in the compressive epilayers $\left(\sigma_{\mathrm{HH}} / \sigma_{\mathrm{LH}} \approx 2.8\right)$. This apparent relationship between microscopical inhomogeneities and the sign of strain seems rather general and should be analyzed further. The values of $\epsilon_{x}$ obtained here ranged from 0.6 to $1.5 \mathrm{meV}$; the precise origin of the variation of $\epsilon_{x}$ remains an open question, and for the purposes of this study is considered to be a phenomenological parameter.

In the following subsections we present a procedure for the determination of epilayer strain, alloy composition, and relaxation from the values of $E_{g}$ and $\Delta E$ obtained from the $O A$ spectra. The relative accuracy of this method is then assessed by comparison with DCXD analysis.

\section{A. Determination of the in-plane strain}

It is now well understood that strain raises the degeneracy at the top of the valence band. The HH and LH valence-band energies relative to the conduction band change with strain according to $0^{5,6}$

$$
\Delta E_{\mathrm{HH}}=\Delta_{\mathrm{HH}} e_{11}
$$

and

$$
\Delta E_{\mathrm{LH}}=\Delta_{\mathrm{LH}} e_{11},
$$

where

$$
\Delta_{\mathrm{HH}}=\left(-2 a \frac{\left(c_{11}-c_{12}\right)}{c_{11}}-b \frac{\left(c_{11}+2 c_{12}\right)}{c_{11}}\right)
$$

and

$$
\Delta_{\mathrm{LH}}=\left(-2 a \frac{\left(c_{11}-c_{12}\right)}{c_{11}}+b \frac{\left(c_{11}+2 c_{12}\right)}{c_{11}}\right) .
$$

Here $c_{11}$ and $c_{12}$ are the elastic coefficients and $a$ and $b$ are the hydrostatic and shear deformation potentials, respectively. $e_{11}$ is related to the band-gap splitting energy $\Delta E\left(=E_{\mathrm{gLH}}-E_{\mathrm{gHH}}\right)$ by

$$
\Delta E=\left(\Delta_{\mathrm{HH}}-\Delta_{\mathrm{LH}}\right) e_{11} .
$$

The values of the elastic stiffness coefficients and deformation potentials for InAs and GaAs are shown in Table II. ${ }^{7}$ By linear interpolation, the values of $c_{11}, c_{12}, a$, and $b$ may be deduced for $\operatorname{In}_{x} \mathrm{Ga}_{1-x} \mathrm{As}$, the values of $\Delta_{\mathrm{HH}}(x)$ and $\Delta_{\mathrm{l} H}(x)$ may then be obtained by applying Eq. (5) and (6). However, following this assumption, the variations of both $\Delta_{\mathrm{HH}}$ and $\Delta_{\mathrm{LH}}$ with $x$ are very nearly linear, and therefore may be approximated, with a discrepancy of less than $1 \%$, by

$$
\Delta_{\mathrm{HH}}(x)=9.04 x+12.74(1-x)
$$

and

$$
\Delta_{\mathrm{LH}}(x)=1.53 x+6.26(1-x) \text {. }
$$

By combining Eqs. (7), (8), and (9), the epilayer strain may be calculated directly from the valence-band splitting. At first glance it would appear that without accurate knowledge of the alloy composition the value of strain cannot be calculated. However, the variation of $\Delta_{\mathrm{HH}}, \Delta_{\mathrm{LH}}$, and $\left(\Delta_{\mathrm{HH}}-\Delta_{\mathrm{LH}}\right)$ with $x$ is relatively small, so that only an approximate value of $x$ is needed. An iterative procedure could be used if necessary, although this is not usually the case.

It is necessary at this point to consider the effect of measurement temperature on the band-gap splitting observed. In Fig. 3 the change in the value of $\Delta E$ compared to the value obtained at $4 \mathrm{~K}$ is plotted as a function of the sample temperature. A clear decrease in the splitting is evident as the sample temperature rises in all the samples examined. A possible explanation for this lies in the difference in the thermalexpansion coefficients of the substrate and the epilayer, which alters the strain in the epilayer as the measurement temperature is varied. The significance of this phenomenon may be estimated by integrating,

$$
\Delta e_{11}=\int_{4 \mathrm{~K}}^{T}\left(\alpha_{\mathrm{InGaAs}}-\alpha_{\mathrm{InP}}\right) d T,
$$

where $\alpha_{\mathrm{InGaAs}}$ and $\alpha_{\mathrm{InP}}$ are the thermal-expansion coefficients of the epilayer and substrate, respectively, and $T(\mathrm{~K})$ is the sample temperature. Using the temperature-dependent values

TABLE II. Elastic stiffness coefficients $c_{11}$ and $c_{12}$, and hydrostatic and shear deformation potentials $a$ and $b$ for InAs and GaAs (Ref. 7).

\begin{tabular}{lcccc}
\hline \hline & $c_{\mathrm{l} 1}\left(\times 10^{11} \mathrm{~cm}^{-2}\right)$ & $c_{12}\left(\times 10^{11} \mathrm{~cm}^{-2}\right)$ & $a(\mathrm{eV})$ & $b(\mathrm{eV})$ \\
\hline InAs & 8.329 & 4.526 & -5.79 & -1.8 \\
$\mathrm{GaAs}$ & 11.880 & 5.380 & -8.68 & -1.7 \\
\hline
\end{tabular}




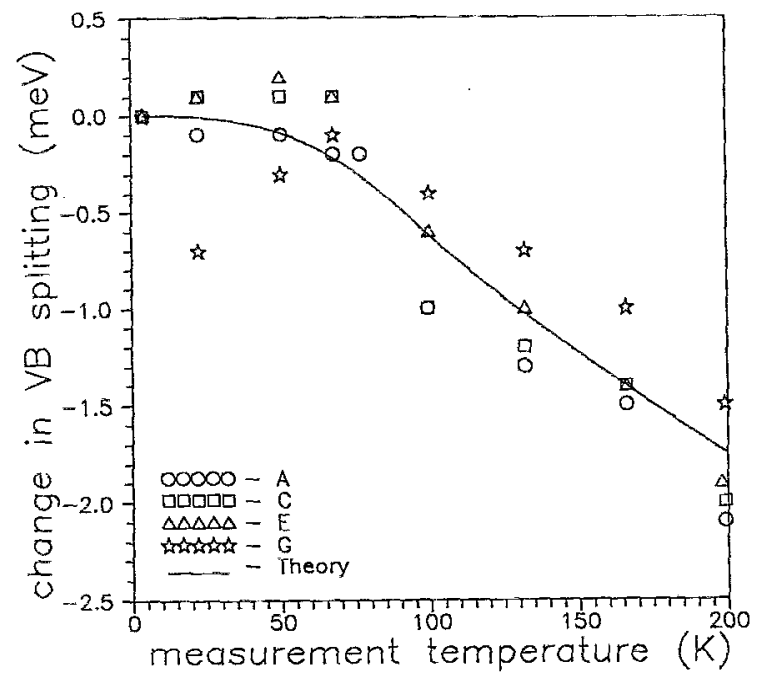

FIG. 3. The experimentally observed change in valence-band splitting as a function of measurement temperature for some of the samples studied. The continuous line shows the evolution of splitting calculated from changes in strain due to the differences in the thermal-expansion coefficients of In $_{53} \mathrm{Ga}_{47}$ As and InP.

of $\alpha$ for InAs, GaAs, and InP in Ref. 8, the additional strain incorporated in an $\mathrm{In}_{53.2} \mathrm{Ga}_{46.8}$ As epilayer by cooling the sample from 300 to $4 \mathrm{~K}$ will be $\Delta e \simeq 0.28 \times 10^{-3}$. The theoretical change in valence-band splitting corresponding to this change in $e_{11}$, calculated from Eq. (7) for an $\operatorname{In}_{53.2} \mathrm{Ga}_{46.8} \mathrm{As}$ epilayer, is also plotted as a function of temperature in Fig. 3. There is reasonable agreement between the theoretical and experimentally observed change in the splitting with measurement temperature. This serves to illustrate the sensitivity of the OA technique to small changes in strain and also indicates that for any comparison. with values of strain measured by DCXD (at $300 \mathrm{~K}$ ), the effect of thermal contraction should be taken into account. Clearly, the ideal solution would be to use the same sample temperature for both techniques. However, the use of higher temperatures for OA increases the energetic dispersion of the spectra, thereby reducing the accuracy of the determination of $E_{g}$ and $\Delta E$. The values of $e_{11}$ measured by DCXD may be extrapolated to 4 $\mathrm{K}$ using the values of the binary lattice constants shown in Table III. In Fig. 4 these values of $e_{11}$ are plotted against the equivalent values of $e_{11}$ measured by $\mathrm{OA}$. Over the range examined, good quantitative agreement is evident, and provided that the effects of the different thermal-expansion coefficients are taken into account, the discrepancies observed between both techniques do not exceed $\Delta e_{11}= \pm 0.27 \times 10^{-3}$.

TABLE III. Lattice constants of InP, GaAs, and InAs at 300, 77, and $4 \mathrm{~K}$. Values at $300 \mathrm{~K}$ are from Ref. 7; 77 and $4 \mathrm{~K}$ values were calculated from Eq. (10), using values of $\alpha(T)$ from Ref. 8 .

\begin{tabular}{|c|c|c|c|}
\hline Temperature $(\mathrm{K})$ & $a_{\operatorname{InP}}(\ddot{A})$ & $a_{\mathrm{GaAs}}(\AA)$ & $a_{\operatorname{InAs}}(\mathrm{A})$ \\
\hline 300 & 5.86875 & 5.65315 & 6.05838 \\
\hline $77 \ldots$ & 5.86530 & 5.64813 & 6.05389 \\
\hline 4 & 5.86571 & 5.64816 & 6.05401 \\
\hline
\end{tabular}

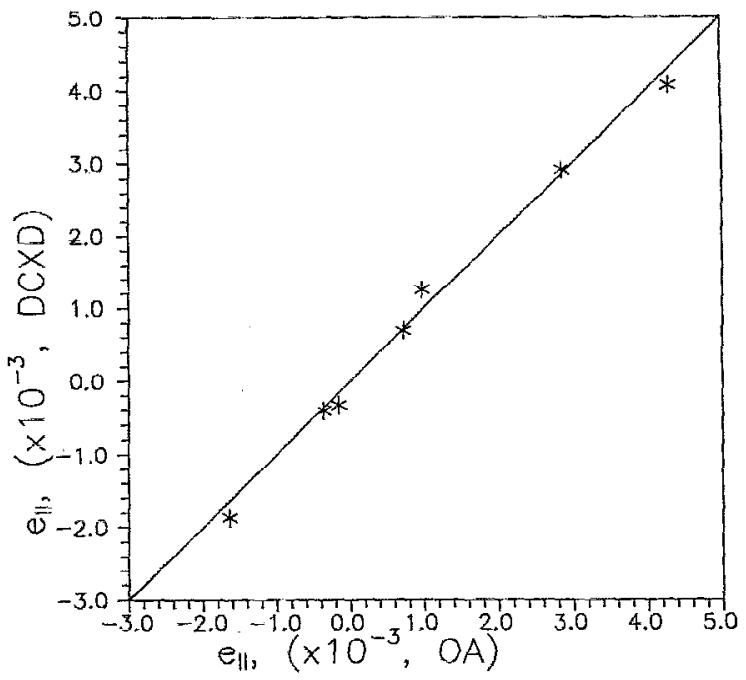

FIG. 4. Comparison of values of strain obtained by $O A$ and DCXD. OA measurements were performed at $4 \mathrm{~K}, \mathrm{DCXD}$ values were extrapolated to 4 $\mathrm{K}$ using the lattice constant values given in Table II.

From Eq. (7), this discrepancy is equivalent to a valenceband splitting of $\approx \pm 1.9 \mathrm{meV}$. The accuracy of the determination of $\Delta E$ is estimated to be better than $\pm 0.3 \mathrm{meV}$, indicating that the uncertainty in the determination of $e_{11}$ does not originate solely from limitations in the determination of $\Delta E$. Linear interpolation from the binary values of elastic stiffness constants and deformation potentials may introduce some uncertainty to the calculation of $e_{11}$. In fact, in $\mathrm{In}_{x} \mathrm{Ga}_{1-x} \mathrm{As}_{y} \mathrm{P}_{1-y}$ materials it is known that these parameters do not follow a linear dependence with $x$ and $y ;{ }^{9}$ however, if for $\operatorname{In}_{x} \mathrm{Ga}_{1-x}$ As the lack of linearity were significant in the calculation of $\Delta_{\mathrm{HH}}$ and $\Delta_{\mathrm{LH}}$, a systematic variation between the values of strain measured by DCXD and OA would be observed. Over the range of alloy compositions examined this is not the case, indicating that linear interpolation is valid.

\section{B. Determination of alloy composition}

For unstrained $\operatorname{In}_{x} \mathrm{Ga}_{1-x}$ As layers, the band-gap energy $E_{g 0}$ depends on the molar composition of the epilayer; at $4 \mathrm{~K}$ this is given by ${ }^{7}$

$$
E_{g 0}(x)=1.519-1.608 x+0.5 x^{2}(\mathrm{eV}) .
$$

For a compressively strained epilayer the band-gap value $E_{g}$ is modified by an amount $\Delta E_{L H}$, so that

$$
E_{g}=E_{g 0}-\Delta E_{\mathrm{LH}},
$$

and for epilayers subject to tensile strain

$$
E_{\mathrm{g}}=E_{80}-\Delta E_{\mathrm{FH}} \text {. }
$$

Thus the alloy composition of a strained epilayer may be deduced from the experimental measurements of the valence-band splitting and the band gap $E_{g}$. In Fig. 5 the values of $x$ obtained in this way from OA spectra are compared with the values of $x$ obtained by DCXD; once again there is a good agreement, with the maximum observed dis- 


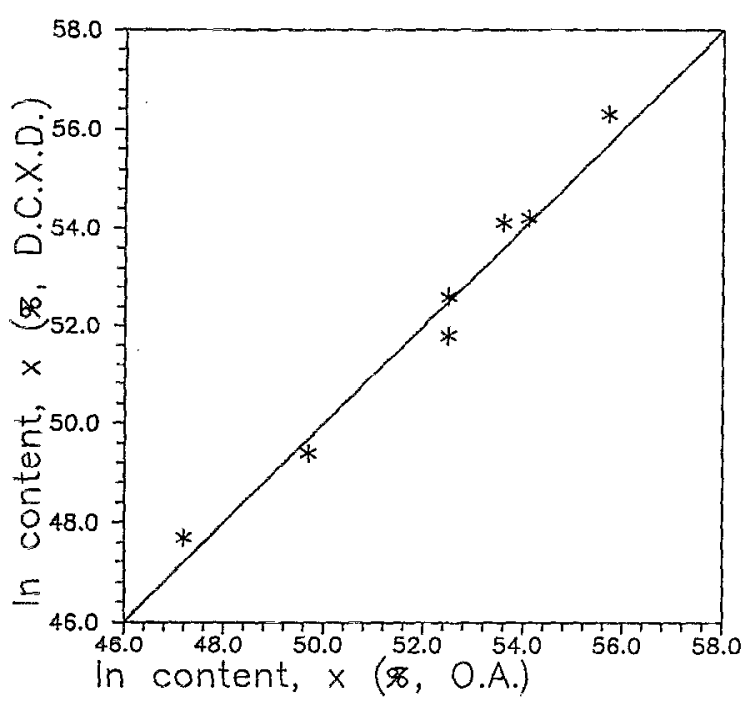

FIG. 5. Comparison of the values of alloy composition obtained by $\mathrm{OA}$ and DCXD.

crepancy between the two techniques $\Delta x= \pm 0.7 \%$. From the differential of Eq. (11) it can be shown that the equivalent of this discrepancy in terms of energy is $\approx \pm 15.0 \mathrm{meV}$, yet it is estimated that $E_{g}$ can be measured by $\mathrm{OA}$ with an accuracy of $\approx \pm 2.0 \mathrm{meV}$. Thus, as for measurements of $e_{11}$, uncertainties in the measurement of $x$ cannot be attributed solely to random experimental errors in the $\mathrm{OA}$ technique. The precise cause of these discrepancies and of those in the measurement of $e_{11}$ is an open question, although they may indicate that: (i) additional mechanisms are involved in the relationship between $e_{11}, x, E_{g}$, and $\Delta E$; (ii) the strain is not perfectly biaxial; or (iii) there are inaccuracies in the DCXD technique.

\section{Measurement of relaxation}

As epilayer growth proceeds a critical thickness is eventually reached, beyond which the formation of dislocations is energetically favorable to the maintenance of complete registry between the substrate and the epilayer. The degree of relaxation $\eta$ may be expressed as

$$
\eta=e_{11} / e_{\max }
$$

where $e_{\max }$ is the strain prior to relaxation $\left[e_{\max }=\left(a_{\mathrm{InP}}-a_{0}\right) / a_{0}\right]$. Thus, for a pseudomorphic epilayer $\eta=1$ and for a completely relaxed epilayer $\eta=0$. On this basis the values of $\eta$ measured by OA for the samples examined in this study are shown in Table IV; the degree of relaxation was too small to be quantified by DCXD (i.e., $\eta \approx 1$ ). With the exception of sample $E$, the $O A$ measurements of $\eta$ were near unity, but some samples have anomalous values of $\eta>1$. From Eq. (14), the error in the measurement of relaxation $\Delta \eta$ is given by

$$
\Delta \eta=\left(\frac{\Delta e_{11}}{e_{\max }}+\frac{\Delta e_{\max } e_{11}}{e_{\max }^{2}}\right) .
$$

The maximum possible error in $\eta$ may be estimated by assuming that the discrepancies between measurements of
TABLE IV. Values of lattice relaxation $\eta$ and alloy composition $x$ deduced from OA measurements.

\begin{tabular}{ccc}
\hline Sample & $\eta$ & $\begin{array}{c}\text { In content } x \\
(\mathrm{OA}, \%)\end{array}$ \\
\hline A & 0.97 & 47.2 \\
B & 1.06 & 49.7 \\
C & 1.27 & 52.5 \\
D & 0.94 & 52.5 \\
E & 1 & 53.6 \\
F & 1.07 & 54.1 \\
G & 1.13 & 55.7 \\
\hline
\end{tabular}

strain and alloy composition by DCXD and OA arise solely from limitations inherent to the $O A$ technique. Using the value of $\Delta e_{11}$ from Sec. IV a, and calculating $\Delta e_{\max }$ from $\Delta x$ (Sec. IV b), we have

$$
\Delta \eta=\left(\frac{0.27 \times 10^{-3}}{e_{\max }}+\frac{0.48 \times 10^{-3} e_{11}}{e_{\max }^{2}}\right) .
$$

On this basis, in Fig. 6 the maximum possible error in the measurement of $\eta$ is plotted as a function of alloy composition. As expected, in common with other techniques including $\mathrm{DCXD}$, the error in the measurement of $\eta$ increases as the values of $e_{11}$ and $e_{\max }$ are reduced, i.e., as lattice-matched conditions are approached, which may account for the unusually large value of $\eta$ deduced for sample $\mathrm{E}$. It is worth noting here that $e_{\max }$ and $x$ were interrelated using the values of binary lattice constants at $4 \mathrm{~K}$, corresponding to the OA measurement temperature: Thus, the value for lattice match to InP indicated is $x=53.6 \%$, (i.e., the value of $x$ for sample E). The individual cases of substrate/epilayer coherency $(\eta$ $=1$ ), partial relaxation $(\eta=0.5)$ and complete relaxation

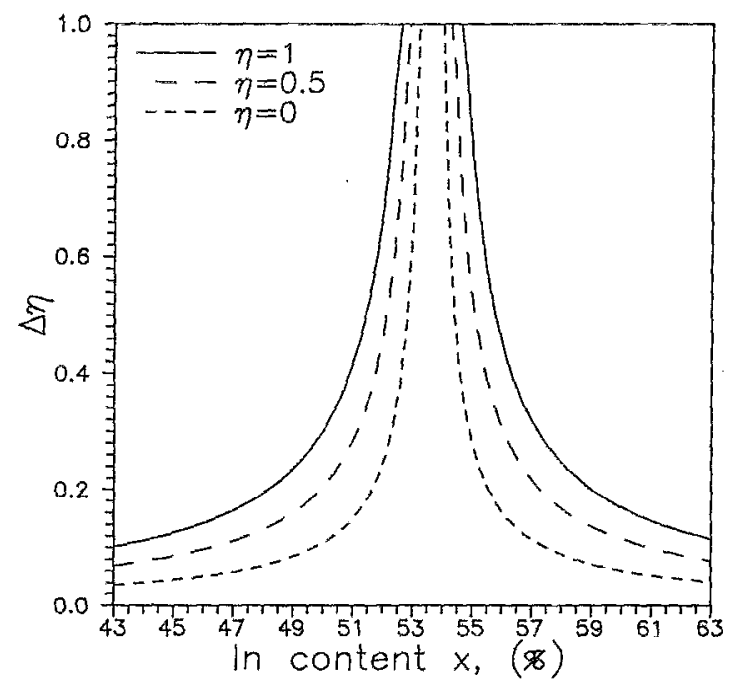

FIG. 6. Estimated maximum error in the measurement of relaxation $\eta$, as a function of alloy composition for $\operatorname{In}_{x} \mathrm{Ga}_{1-x}$ As on $\mathrm{InP}$, by $O A$, deduced from Eq. (16). The solid lines denote the situation for unrelaxed layers $(\eta=1)$, the longer dashed lines for partially relaxed layers $(\eta=0.5)$, and the shorter dashed lines for fully relaxed layers $(\eta=0)$. 
$(\eta=0)$ are considered, showing that for a particular value of $x$, uncertainties in the measurement of $\Delta \eta$ decrease as the layer relaxes.

\section{DISCUSSION}

Our results show that OA may be used independently to determine strain, alloy composition, and relaxation in $\mathrm{In}_{x} \mathrm{Ga}_{1-x}$ As epilayers. Other optical and $\mathrm{x}$-ray techniques are capable of measuring the same parameters in mismatched heterostructures and so it is worth considering the limitations of the technique and also the advantages over other methods.

$O A$ is a relatively straightforward technique, requiring the measurement of only one spectrum per sample to obtain all three parameters, whereas similar characterization by DCXD requires the measurement of several rocking curves. Furthermore, it has been shown that $O A$ can also provide a simple method for the identification and quantification of microscopic inhomogeneities in $\mathrm{In}_{x} \mathrm{Ga}_{1-x}$ As. ${ }^{4,10}$ It is capable of clearly resolving valence-band splitting, to the extent that changes resulting from thermal contraction may be measured. In photoluminescence spectroscopy the signal depends on the thermal occupation of the states. At low temperature it is impossible to measure splitting because the upper state is not occupied. Higher temperatures must then be used to populate the upper band, at the expense of resolution. Raman scattering is also capable of measuring strain in $\operatorname{In}_{x} \mathrm{Ga}_{1-x} \mathrm{As}$ layers. This is usually obtained from the shift of the LO GaAs-like mode in the strained layer, in comparison with the unstrained alloy (the InAs-like mode being far less intense). Following this method, assuming an uncertainty in the position of the Raman peaks of $0.1 \mathrm{~cm}^{-1}$, the minimum strain that can be detected is $e_{11} \approx 0.2 \times 10^{-3}{ }^{11}$ This is similar to the dispersion in the values of strain measured by $\mathrm{OA}$ and DCXD. However, to measure strain by Raman scattering either the alloy composition must be known, or similar measurements must be performed on an unstrained (i.e., thick) epilayer of the same composition. Furthermore, in addition to strain, the position of the Raman peaks may also be altered by disorder, if a high density of structural defects is present in the layer.

One major limitation of the OA technique is that it cannot by used for heterostructures where the band gap of the substrate is smaller than the epilayer; however, in this case an OA-like spectrum may be obtained by photoluminescence excitation spectroscopy (PLE). Another limitation is epilayer thickness, the minimum depends on the material quality and the spectrometer used. The thinnest $\mathrm{In}_{x} \mathrm{Ga}_{1-x} \mathrm{As}$ layers we have measured so far were $0.1 \mu \mathrm{m}$ thick.

\section{CONCLUSIONS}

We have assessed, for the first time, the validity of the OA technique for the measurement of structural parameters in strained $\mathrm{In}_{x} \mathrm{Ga}_{1-x} \mathrm{As} / \mathrm{InP}$ epilayers. Provided that strain arising from the different temperatures employed in the two techniques is accounted for, measurements of strain by $\mathrm{OA}$ show good agreement with DCXD results, with a dispersion in the values of strain obtained by the two techniques of $\Delta e_{11}= \pm 0.27 \times 10^{-3}$, over the range examined. Similar analysis of alloy composition measurements has shown a dispersion in the values of $x$ of $\Delta x= \pm 0.7 \%$. The extent of lattice relaxation may also be determined by the OA technique. The degree of uncertainty in this parameter increases as lattice match is approached and is also greater for unrelaxed layers.

\section{ACKNOWLEDGMENT}

This study was supported, in part, by the European Community under the Human Capital Mobility Scheme, Contract No. ERBCHBGCT920029.

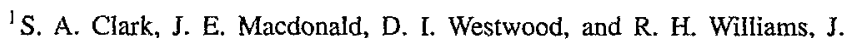
Cryst. Growth 121, 743 (1992).

${ }^{2}$ T. C. Chong and C. G. Fonstad, IEEE J. Quantum Electron. QE-24, 171 (1989).

${ }^{3}$ J. Elliot, Phys. Rev. 108, 1383 (1957).

${ }^{4}$ P. Roura. J. Bosch, and J. R. Morante, Phys. Rev. B 46, 10453 (1992).

${ }^{5}$ F. H. Pollak and M. Cardona, Phys. Rev. 172, 816 (1968).

${ }^{6}$ A. Gavini and M. Cardona, Phys. Rev. B 1, 672 (1970).

${ }^{7}$ D. K. Gaskill, N. Bottka, L. Aina, and M. Mattingly, Appl. Phys. Lett. 56, 1269 (1990).

${ }^{8}$ Data in Science and Technology-Semiconductors, edited by O. Madelung (Springer, Berlin, 1991).

"S. Adachi, J. Appl. Phys. 53, 8775 (1982).

${ }^{10}$ P. Roura, J. Bosch, S. A. Clark, F. Peiro, A. Cornet, J. R. Morante, and R. H. Williams (unpublished).

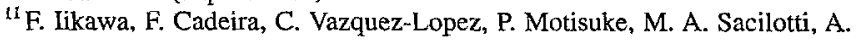
P. Roth, and R. A. Masut, Solid State Commun. 68, 211 (1988). 